\title{
Phonons in Intrinsic Josephson Systems with Parallel Magnetic Field
}

\author{
C. Preis ${ }^{\mathrm{a}}$, C. Helm ${ }^{\mathrm{b}}$, K. Schmalzl ${ }^{\mathrm{a}}$, J. Keller ${ }^{\mathrm{a}}$, R. Kleiner ${ }^{\mathrm{c}}$, P. Müller ${ }^{\mathrm{d}}$ \\ anstitut für Theoretische Physik, Universität Regensburg, D-93040 Regensburg, Germany \\ ${ }^{\mathrm{b}}$ Los Alamos National Laboratory, T-11, Los Alamos, NM 87545, USA \\ cPhysikalisches Institut, Universität Tübingen, D-72076 Tübingen, Germany \\ dPhysikalisches Institut III, Universität Erlangen-Nürnberg, D-91058 Erlangen, Germany
}

Subgap resonances in the $I$ - $V$ curves of layered superconductors are explained by the coupling between Josephson oscillations and phonons with dispersion in c-direction. In the presence of a magnetic field applied parallel to the layers additional structures due to fluxon motion appear. Their coupling with phonons is investigated theoretically and a shift of the phonon resonances in strong magnetic fields is predicted.

PACS: 74.50.+r, 74.60.Ge, Keywords: Josephson, phonons, flux motion

\section{Excitation of phonons by Josephson os- cillations}

The c-axis transport in the highly anisotropic cuprate-superconductors $\mathrm{Tl}_{2} \mathrm{Ba}_{2} \mathrm{Ca}_{2} \mathrm{Cu}_{3} \mathrm{O}_{10+\delta}$ (TBCCO) and $\mathrm{Bi}_{2} \mathrm{Sr}_{2} \mathrm{CaCu}_{2} \mathrm{O}_{8+\delta}$ (BSCCO) can well be described by a model where the superconducting $\mathrm{CuO}_{2}$ layers are coupled by tunneling barriers forming a stack of Josephson junctions [1.2].

Subgap structures [3] in the $I-V$ characteristics could be explained by the excitation of longitudinal c-axis phonons by the Josephson oscillations in resistive junctions [5] 7]. In Ref. [8] a microscopic theory for the coupling between Josephson oscillations and phonons has been derived for the case of a short stack of Josephson junctions where it can be assumed that the gaugeinvariant phase difference $\gamma_{n}(x, t)=\varphi_{n}(x, t)-$ $\varphi_{n+1}(x, t)-\frac{2 e}{\hbar} \int_{n}^{n+1} d z A_{z}(x, z, t)$ between superconducting layers $n$ and $n+1$ is constant along the layers (independent of $x$ ). In the simplest model the tunneling current through a junction $n$ is relatexd the bias current density $j_{b}$ by the RSJ equation

$j_{b}=j_{c} \sin \gamma_{n}(t)+\sigma E_{n}(t)+\dot{D}_{n}(t)$.

Here $E_{n}(t)$ is the average electric field across the junction which is related to the phase differ- ence $\gamma_{n}$ by the second Josephson equation $\hbar \dot{\gamma}_{n}=$ $2 e d E_{n}$ where $d$ is the thickness of the barrier.

$D_{n}=E_{n}^{\rho}$ is the field generated by the oscillating conduction electron charges on the $\mathrm{CuO}_{2}$ layers. It can be expressed by the average field in the barrier and the ionic polarization, $D_{n}=$ $\epsilon_{0} E_{n}+P_{n}$. The polarization $P_{n}$ is proportional to the lattice displacements of ions. Phonons are excited by the field of the oscillating electronic charges on the $\mathrm{CuO}_{2}$ layers. The field $D\left(k_{z}\right)$ can be expressed by a generalized dielectric function $D\left(k_{z}\right)=\epsilon_{0} \epsilon_{z z}^{\mathrm{ph}}\left(k_{z}, \omega\right) E\left(k_{z}\right)$ of the form

$\epsilon_{z z}^{\mathrm{ph}}\left(k_{z}, \omega\right)=\left(1-\sum_{\lambda} \frac{\left|\Omega\left(k_{z}, \lambda\right)\right|^{2}}{\omega^{2}\left(k_{z}, \lambda\right)-\omega^{2}}\right)^{-1}$.

Here $\omega\left(k_{z}, \lambda\right)$ are the eigenfrequencies of the dynamical matrix (including long-range Coulomb forces). The oscillator strength $\left|\Omega\left(k_{z}, \lambda\right)\right|^{2}$ takes care of the fact that ions inside the barrier and on the $\mathrm{CuO}_{2}$ layers are excited by different fields and have to be counted differently in the polarization in the RSJ-equation (1).

The dielectric function has zeros at the frequencies of longitudinal c-axis phonons. It can also be written in the more common form

$\epsilon_{z z}^{\mathrm{ph}}\left(k_{z}, \omega\right)=\epsilon_{\infty}+\sum_{\lambda} \frac{\left|\tilde{\Omega}\left(k_{z}, \lambda\right)\right|^{2}}{\tilde{\omega}^{2}\left(k_{z}, \lambda\right)-\omega^{2}}$

where we have included a background DK. The 
frequencies $\tilde{\omega}\left(k_{z}, \lambda\right)$ where the dielectric function has poles can also be calculated from a dynamical matrix where long-range Coulomb forces have been subtracted. In the limit $k_{z} \rightarrow 0$ they correspond to the transversal optical eigenfrequencies of the system.

In the resistive state the phase difference of barrier $n$ has the form

$\gamma_{n}(t)=\theta_{n}+\omega t+\delta \gamma_{n}(t)$

where $\delta \gamma_{n}(t)$ oscillates with the same Josephson frequency $\omega$, while for a barrier in the superconducting state the term $\omega t$ is missing. Inserting this ansatz in the RSJ equations we can solve for the dc part and the oscillating part. In the special case where only one junction of the stack is in the resistive state we obtain for the normalized dccurrent $J_{b}:=j_{b} / j_{c}$ as function of the dc-voltage $V=\hbar \omega /(2 e)$ :

$J_{b}=J_{q p}(V)+\frac{\omega_{J}^{2}}{2 \omega^{2}} \frac{\bar{\epsilon}_{2}+\sigma /\left(\epsilon_{0} \omega\right)}{\bar{\epsilon}_{1}^{2}+\left(\bar{\epsilon}_{2}+\sigma /\left(\epsilon_{0} \omega\right)\right)^{2}}$.

Here $\bar{\epsilon}(\omega)=\bar{\epsilon}_{1}(\omega)+i \bar{\epsilon}_{2}(\omega)$ is an averaged phonon dielectric function defined by

$\bar{\epsilon}(\omega)=G^{-1}(\omega)+\frac{\bar{\omega}_{J}^{2}}{\omega^{2}}-\frac{i \sigma}{\epsilon_{0} \omega}$,

$G(\omega)=\frac{1}{N_{z}} \sum_{k_{z}} \frac{1}{\epsilon_{z z}^{\mathrm{ph}}\left(k_{z}, \omega\right)-\frac{\bar{\omega}_{J}^{2}}{\omega^{2}}+\frac{i \sigma}{\epsilon_{0} \omega}}$.

Here $\bar{\omega}_{J}^{2}=\omega_{J}^{2} \sqrt{1-\left(j / j_{c}\right)^{2}}$ and $\omega_{J}^{2}=2 e d j_{c} /\left(\hbar \epsilon_{0}\right)$ is the bare Josephson plasma frequency. In the case of a dispersionless phonon we just have $\bar{\epsilon}(\omega)=\epsilon_{z z}^{\mathrm{ph}}(\omega)$.

In the general case the $I$ - $V$ curve shows peaks at the zeros of the real part of $\bar{\epsilon}(\omega)$ corresponding to c-axis phonons with a high density of states and non-vanishing oscillator strength $|\Omega|^{2}$. Besides optical $\Gamma$-point phonons also phonons from the edge of the Brillouin zone at $k_{z}=\pi / d$ and even acoustical phonons may contribute to phonon resonances in the $I$ - $V$ curves $[8]$.

The coupling to an acoustical phonon at $k_{z}=\pi / d$ may explain a resonance observed for TBCCO at $3.2 \mathrm{mV}$ by Seidel et al. [9] at a frequency/voltage which is lower then any optical phonon branch expected from model calculations.
A fit to the experimental data can be made with reasonable values of the oscillator strength and phonon damping which are compatible with optical experiments. Also a double peak structure found in BSCCO [7] may be due to the coupling to one optical branch with two van Hove singularities at $k_{z}=0$ and $k_{z}=\pi / d$.

The dispersion of phonons also leads to a coupling (phase-locking) of Josephson oscillations in different resistive junctions [8,13]. A phaselocking in a stack of Josephson junctions is important for applications of such systems as highfrequency mixers and detectors.

\section{Vortex motion and phonons}

In the case of long junctions and in the presence of an external magnetic field applied parallel to the layers we have to account for the variation of the phase $\gamma_{n}(x, t)$ along the layers (x-direction). The result is a generalization of the coupled sineGordon equations derived in 10 12]:

$$
\begin{aligned}
& \partial_{x}^{2} \gamma_{n}(x, t)=\frac{1}{\lambda_{J}^{2}} J_{n}-\frac{1}{\lambda_{K}^{2}}\left(J_{n+1}+J_{n-1}\right) \\
& J_{n}=\sin \gamma_{n}+\frac{\sigma}{\epsilon_{0} \omega_{J}^{2}} \dot{\gamma}_{n}+\frac{1}{\omega_{J}^{2}} \epsilon_{z z}^{\mathrm{ph}} \ddot{\gamma}_{n} .
\end{aligned}
$$

The characteristic lengths for the variation of the phase along the layers are calculated from

$$
\frac{1}{\lambda_{K}^{2}}=\frac{d^{2}}{\lambda_{c}^{2} \lambda_{a b}^{2}} ; \quad \frac{1}{\lambda_{J}^{2}}=\frac{1}{\lambda_{c}^{2}}+\frac{2}{\lambda_{K}^{2}}
$$

where $\lambda_{c}, \lambda_{a b}$ are the penetration depths of magnetic fields polarized in c-direction and parallel to the ab-planes, respectively. They are related to the corresponding plasma frequencies $\lambda_{c}=c / \omega_{J}$, $\lambda_{a b}=c / \omega_{a b}$, where $c$ is the velocity of light. For BSCCO realistic values are $\lambda_{a b}=170 \mathrm{~nm}$, $d=1.5 \mathrm{~nm}, \lambda_{c}=150 \mu \mathrm{m}$, which gives a Josephson penetration depth of $\lambda_{J} \simeq 1 \mu \mathrm{m}$.

In the derivation of Eq. (8) terms of the form $\ddot{\gamma} / \omega_{a b}^{2}$, which are small compared to $\gamma$ for the frequency range considered, have been neglected. Furthermore in the coupling to phonons only polarization effects with polarization in c-direction excited by fields in c-direction (expressed by the dielectric function $\left.\epsilon_{z z}^{\mathrm{ph}}\left(k_{x}, k_{z}, \omega\right)\right)$ have been taken 
into account. It can be shown [13], that the neglect of other polarizations is a good approximation for wave-vectors with $k_{x} d \ll 1$, which is well fulfilled for $k_{x} \leq 1 / \lambda_{J}$. On the other hand, $k_{z}$ is not small and $k_{z} \gg k_{x}$ in general.

In the following numerical calculations we consider only local polarization effects, i.e. we neglect the dispersion of phonons. This will be sufficient to demonstrate the principle effects.

\subsection{Boundary conditions}

Assuming a stack with $N$ Josephson barriers with $n=1 \ldots N+1$ superconducting layers and two normal contacts $n=0, N+2$ this set of equations (8) holds for $n=1 \ldots N$. In the barriers connecting the superconducting layers with the normal contact $J_{n}$ has to be replaced by the normalized bias current $J_{b}=j_{b} / j_{c}$. It is useful to incorporate this boundary condition into the set of equations by subtracting $J_{b}$ for each term writing $\tilde{J}_{n}:=J_{n}-J_{b}$ then

$\partial_{x}^{2} \gamma_{n}-\frac{1}{\lambda_{c}^{2}} J_{b}=\frac{1}{\lambda_{J}^{2}} \tilde{J}_{n}-\frac{1}{\lambda_{K}^{2}}\left(\tilde{J}_{n+1}+\tilde{J}_{n-1}\right)$

which has to be solved with the boundary conditions $\tilde{J}_{n}=0$ for $n=0, N+1$ for a finite stack.

The magnetic field (in y-direction) which causes the variation of the phase along the layers enters explicitly the boundary condition for $\partial_{x} \gamma_{n}$ at the edges of the stack. It consists of the external field $B_{\text {ext }}$ and the field generated by the bias current. If we neglect the latter we have

$\partial_{x} \gamma_{n}(x=0)=\partial_{x} \gamma_{n}\left(x=L_{x}\right)=\frac{2 e d}{\hbar} B_{e x t}=: \eta$

and we may drop the small contribution $J_{b} / \lambda_{c}^{2}$ on the left side of Eq. (10). Then in the case of a constant phase along the layers one recovers the RSJ equation $\tilde{J}_{n}=0$.

\subsection{Analytical solution}

The set of coupled sine-Gordon equations can be solved numerically. Here one makes the general ansatz for the phases in the different layers:

$\gamma_{n}(x, t)=\Gamma_{n}(t)+\eta x+\sum_{m=1}^{M} \delta \gamma_{n}(m, t) \cos \left(\frac{m \pi x}{L_{x}}\right)(12)$

using a Fourier expansion for the spatially oscillating part of the phases and splitting off the term $\eta x$ which takes care of the average increase of the phase difference due to the applied magnetic field. With help of this ansatz a coupled set of differential equations for the components $\delta \gamma_{n}(m, t)$ can be derived and solved with Runge-Kutta techniques.

Approximate analytical solutions are possible by setting

$\Gamma_{n}(t)=\theta_{n}+\omega t+\delta \gamma_{n}(0, t)$

and linearizing the equations (8) with respect to small oscillating terms $\delta \gamma_{n}(m, t)$. This ansatz is well justified for large magnetic fields where the magnetic flux penetrates the stack almost homogeneously. Therefore there is a voltage drop $V_{n}=$ $\left\langle\dot{\gamma}_{n}(t)\right\rangle \hbar /(2 e)=\omega \hbar /(2 e)$ over each junction. The oscillating part describes standing waves which oscillate primarily with the same basic Josephson frequency $\omega$.

Using in addition a Fourier expansion of the oscillating parts in c-direction and keeping only the lowest harmonics in $\omega$ we arrive at the following expression for the dc-current

$$
J_{b}=\frac{\omega \sigma}{\epsilon_{0} \omega_{\mathrm{J}}^{2}}-2 \frac{\omega_{\mathrm{J}}^{2}}{\omega^{2}} \frac{1}{N^{2}} \sum_{k_{x}, k_{z}} \operatorname{Im}\left(P\left(k_{x}, k_{z}, \omega\right)\right)
$$

with

$$
P\left(k_{x}, k_{z}, \omega\right)=\frac{\left|I\left(k_{x}, \eta\right)\right|^{2}\left|p\left(k_{z}\right)\right|^{2}}{\epsilon_{z z}^{\mathrm{ph}}\left(k_{x}, k_{z}, \omega\right)+i \frac{\sigma}{\epsilon_{0} \omega}-\frac{k_{x}^{2} \tilde{c}^{2}\left(k_{z}\right)}{\omega^{2}}}
$$

where the sum goes over the discrete values of $k_{x}=m \pi / L_{x}, k_{z}=n \pi /((N+1) d), 0<|n| \leq N$.

The denominator contains the phonon dielectric function and the characteristic velocity

$\tilde{c}\left(k_{z}\right)=c / \sqrt{1-2 \frac{\lambda_{a b}^{2}}{d^{2}}\left(\cos \left(k_{z} d\right)-1\right)}$.

Resonances are expected at frequencies where the real part of the denominator vanishes. There size depends on the weighting functions

$p\left(k_{z}\right)=\sum_{n} e^{-i \theta_{n}} e^{-i k_{z} n}$

which contains a Fourier transformation of the static phase distribution and the function

$I\left(k_{x}, \eta\right)=\frac{i}{2 L_{x}} \int_{0}^{L_{x}} d x e^{-i \eta x} e^{i k_{x} x}$ 
depending on the magnetic field, which is peaked at $k_{x} \simeq \eta$.

\section{Single contact in a magnetic field}

For a single contact, $N=1$, the value of $k_{z}$ is fixed to $k_{z}=\pi /(2 d)$. Then the characteristic (Swihart-) velocity is $\tilde{c}=c_{0} / \sqrt{1+2 \lambda_{a b}^{2} / d^{2}}=$ $\omega_{J} \lambda_{J}$, and the resonance frequencies in the $I-V$ curve are determined from

$\operatorname{Re}\left[\epsilon_{z z}^{\mathrm{ph}}(\omega)-\left(\frac{k_{x} \tilde{c}}{\omega}\right)^{2}\right]=0$.

For a constant $\epsilon_{z z}^{\mathrm{ph}}$ structures appear in the current-voltage characteristic at $\omega_{\text {res }, m}=$ $m \pi \tilde{c} /\left(\sqrt{\epsilon_{z z}^{\mathrm{ph}}} L_{x}\right)$. These are the well-known Fiske steps [14]. They correspond to the excitation of standing electromagnetic waves in the Josephson junction of length $L_{x}$. The largest amplitude is obtained for wave-vectors $k_{x} \simeq \eta$. Here the velocity of fluxons equals the phase velocity of the electromagnetic waves. In the case of a very long junction the Fiske steps merge into one flux flow branch which is peaked at $V=\hbar \omega_{\text {res }} /(2 e)=$ $\tilde{c} d B_{\text {ext }} / \sqrt{\epsilon_{z z}^{\mathrm{ph}}}$ (Eck-peak 150).

In order to discuss the influence of phonons we use here for simplicity a dispersionless optical phonon band with the dielectric function

$\epsilon_{z z}^{\mathrm{ph}}(\omega)=\epsilon_{\infty}+\frac{|\Omega|^{2}}{\omega_{\text {TO }}^{2}-\omega^{2}-i r \omega}$.

The spectrum of resonances as function of the discrete values of $k_{x}=m \pi / L_{x}$ is shown in Fig. 1. One obtains two branches: for small $k_{x}$-values the lower branch corresponds to the propagation of electromagnetic waves, while the upper branch is phonon-like. The lower branch ends at the (transverse) phonon eigenfrequency $\omega_{\mathrm{TO}}$. The upper branch starts at the zero of the dielectric function, i.e. at the longitudinal eigenfrequency $\omega_{\mathrm{LO}}$. The parameters used to calculate the dispersion shown in Fig. 1 are adapted to TBCCO.

In Fig. 2 the result for the $I-V$ curve is shown for three different magnetic fields. The figures compare numerical (Runge-Kutta) with analytical results. Note that the magnetic field selects the $k_{x}$-value where the strongest resonance occurs: $k_{x}^{\max }=\eta=(2 e d / \hbar) B_{\text {ext }}$. In order to show

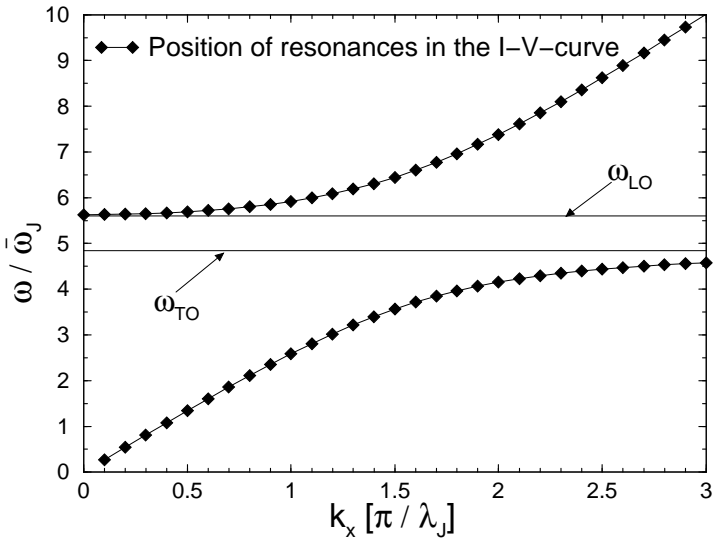

Figure 1. Resonance frequencies for a single Josephson contact with one dispersionless phonon mode.

the phonon effects more clearly a large separation between $\omega_{\mathrm{TO}}$ and $\omega_{\mathrm{LO}}$ has been chosen. Furthermore for numerical reasons a comparatively small value of the McCumber parameter $\beta_{c}=50$ has been used, while $\beta_{c}=500$ would be more realistic $\left(\beta_{c}=\omega_{c}^{2} / \omega_{J}^{2}\right.$ with $\left.\omega_{c}=2 e d j_{c} /(\hbar \sigma)\right)$. For $B_{\text {ext }}=0$ only one resonance occurs at $\omega_{\mathrm{LO}}$ corresponding to the subgap-resonance discussed in Sec. 1. For finite $B_{\text {ext }}$ one finds two groups of resonances corresponding to the two branches in Fig. 1. The fine-structure is due to Fiske-resonances in the stack of finite length $L_{x}$. With increasing field strength the upper peak shifts to higher frequencies. The lower peak approaches the TOfrequency while loosing weight. In all cases there is a gap with no resonances for frequencies between $\omega_{\mathrm{TO}}$ and $\omega_{\mathrm{LO}}$.

Generally the agreement between numerical and analytical calculations is good, in particular, concerning the position of peaks. The agreement in the height of the peaks can be improved by going beyond the linear approximation in the oscillation amplitudes. The numerical calculations show the same hysteretic behavior as the experimental results if the bias current density is changed. 

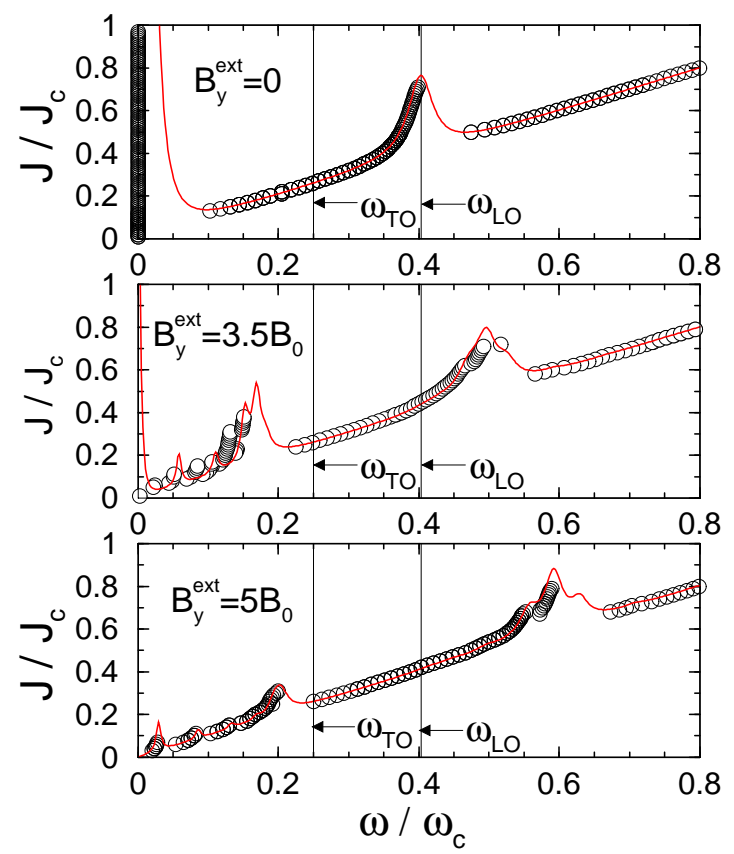

Figure 2. $I-V$ curves for a single contact for three different magnetic fields. $B_{0}=\Phi_{0} /\left(d L_{x}\right)$ corresponds to a magnetic field with one flux quantum per junction.

\section{Several contacts in a magnetic field}

Numerical calculations can also be performed for larger stacks. Analytical calculations are no longer possible for $N>2$ without further assumptions on the relative phases $\theta_{n}$ for the different junctions. In Fig. 3 we show results for the positions of the resonances for a stack with $N=4$ junctions for all possible values of $k_{z}=n \pi /(5 d)$. The multiple branches correspond to different values of the characteristic velocity $\tilde{c}$ which depends strongly on $k_{z}$.

In Fig. 4 we show results for the $I-V$ curve for a large applied magnetic field. Here we find a vortex lattice which is moving due to the bias current. Both numerical and analytical results are shown as function of frequency which corresponds to the dc-voltage drop over a single junction. For

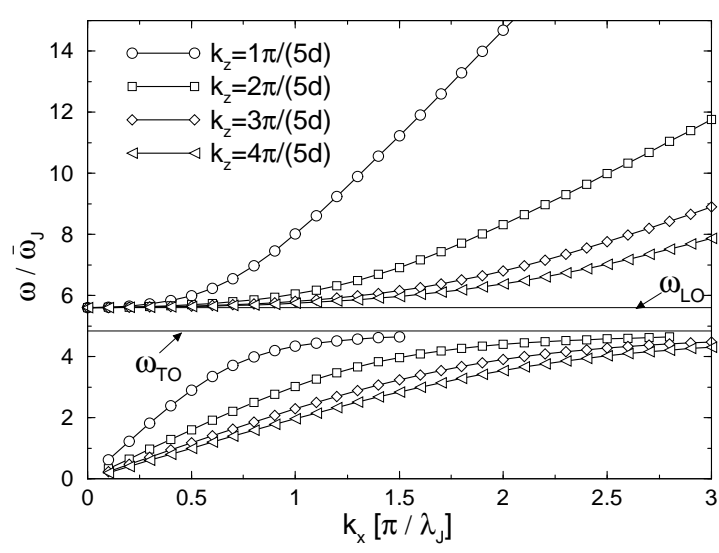

Figure 3. Resonance frequencies for a stack of 4 contacts.

our analytical calculations of the $I$ - $V$ curves we have superimposed results calculated with all possible values of $k_{z}$. The comparison with numerical results in Fig. 4 demonstrates that modes with all possible $k_{z}$-values will be excited by cycling the bias current. The group of peaks at the highest frequency correspond to electromagnetic modes with the smallest $k_{z}$ value. The middle group contains phonon like excitations while the lowest group are electromagnetic excitations of Kleiner modes. Again a gap appears between $\omega_{\text {TO }}$ and $\omega_{\mathrm{LO}}$ in accordance with the dispersion curves in Fig. 3.

\section{Comparison with experimental results and conclusions}

The main results of the preceding section are: a) a shift of phonon resonances in a parallel magnetic field, b) Fiske resonances in the frequency range of phonons are no longer equidistant, c) the flux-flow voltage is no longer proportional to the magnetic field and has a gap between $\omega_{\mathrm{TO}}$ and $\omega_{\mathrm{LO}}$. In order to observe these effects experimentally a strong magnetic field has to be applied $(>3 \mathrm{~T})$. In most experiments [7] the applied field has been much lower, and no shift of phonon resonances has been observed. A further require- 


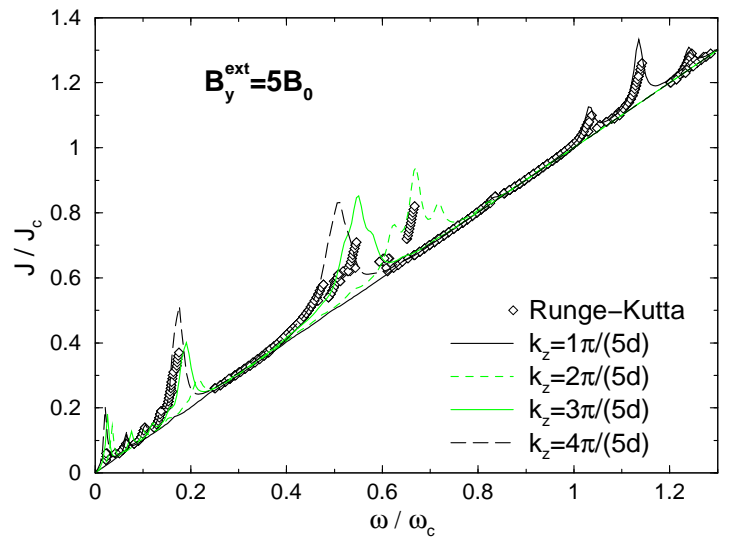

Figure 4. $I-V$ curves for a stack of 4 contacts in high magnetic field.

ment is that the field is applied strictly parallel to the layers in order to avoid vortex pinning from inhomogeneities. The fact that in the cited experiments [4] neither Fiske resonances in the frequency range of phonons nor a flux flow branch could be observed is an indication of vortex pinning. Recently the flux flow voltage has been measured for a stack of 30 junctions in BSCCO [16]. Deviations from the linear field dependence together with anomalies in the frequency range of an optical phonon have been observed, which supports the present model for the interaction between flux flow and phonons.

The author would like to thank K. Schlenga and L. Bulaevskii for fruitful discussions. Financial support by the Bayerische Forschungsstiftung (C.P.) and the Department of Energy under contract W-7405-ENG-36 (C.H.) is gratefully acknowledged.

\section{REFERENCES}

1. R. Kleiner, F. Steinmeyer, G. Kunkel, P. Müller, Phys. Rev. Lett. 68, 2394 (1992)

2. R. Kleiner, P. Müller, Phys. Rev. B 49, 1327 (1994).

3. K. Schlenga, G. Hechtfischer, R. Kleiner, W. Walkenhorst, P. Müller, H.L. Johnson, M.
Veith, W. Brodkorb, E. Steinbeiss, Phys. Rev. Lett. 76, 4943 (1996)

4. A. Yurgens, D. Winkler, N. Zavaritsky, T. Claeson, In: D. Pavuna, I. Bozovic (Eds.), Oxide Superconductor Physics and Nano-Engineering II, Proceedings of SPIE Vol. 2697, 433, (1996).

5. C. Helm, C. Preis, F. Forsthofer, J. Keller, K. Schlenga, R. Kleiner, P. Müller, Phys. Rev. Lett. 79, 737 (1997)

6. C. Helm, C. Preis, F. Forsthofer, J. Keller, K. Schlenga, R. Kleiner, P. Müller, Physica C 293, 60 (1997)

7. K. Schlenga, R. Kleiner, G. Hechtfischer, M. Mößle, P. Müller, C. Helm, C. Preis, F. Forsthofer, J. Keller, H.L. Johnson, M. Veith, E. Steinbeiss, Phys. Rev. B 57, 14518 (1998)

8. C. Helm, C. Preis, C Walter, J. Keller, to be published in Phys. Rev. B 62 (2000)

9. P. Seidel, A. Pfuch, U. Hübner, F. Schmidl, H. Schneidewind, T. Ecke, J. Scherbel, Physica C 293, 49 (1997)

10. S. Sakai, P. Bodin, N.F. Pedersen, J. Appl. Phys. 73, 2411 (1993)

11. R. Kleiner, P Müller, H. Kohlstedt, N.F. Perdersen, S. Sakai, Phys. Rev. B 50, 3942 (1994)

12. L. Bulaevskii, M. Zamora, D. Baeriswyl, H. Beck, J.R. Clem, Phys. Rev. B 50, 12831 (1994)

13. C. Preis, $\mathrm{PhD}$ thesis, and to be published

14. M.D. Fiske, Rev. Mod. Phys. 36,221 (1964)

15. R.E. Eck, D.J. Scalapino, B.N. Taylor, Phys. Rev. Lett. 13, 15 (1964)

16. Yu. I. Latyshev, private communication 